\title{
BIOSSORÇÃO PASSIVA DE CROMO (VI) ATRAVÉS DA MICROALGA Spirulina platensis
}

\author{
Clinei Dal Magro, Maitê Carla Deon, Antônio Thomé, Jeferson Steffanello Piccin e Luciane Maria Colla* \\ Faculdade de Engenharia e Arquitetura, Universidade de Passo Fundo, Campus I, BR 285, Bairro São José, CP 611, 99052-900 \\ Passo Fundo - RS, Brasil
}

Recebido em 8/1/13; aceito em 15/4/13; publicado na web em 1/7/13

\begin{abstract}
PASSIVE BIOSORPTION OF CHROMIUM (VI) USING MICROALGAE Spirulina platensis. Effluents containing toxic metals are dangerous and more economical, efficient and environmentally friendly treatments must be studied, with the biosorption process with microbial biomass constituting an efficient solution. Thus, the ability of Spirulina platensis biomass for removing chromium (VI) using passive and active biosorption was evaluated. Inactive microalgae biomass and synthetic solution containing chromium (VI) were used to evaluate important factors in the process and biomass biosorption ability. Results of the experiments showed that microalgae have potential for biosorption of chromium (VI), attaining removal of $100.39 \mathrm{mg} \mathrm{g}^{-1}$, and that $\mathrm{pH}$ was the variable with the greatest influence on the process.
\end{abstract}

Keywords: toxic metal; passive biosorption; effluent.

\section{INTRODUÇÃO}

A contaminação ambiental proveniente de efluentes industriais tem agravado a situação de degradação do meio ambiente e o equilíbrio dos ecossistemas naturais. Um dos compostos mais preocupantes encontrados nesses efluentes são os metais tóxicos, os quais se apresentam nocivos ao meio ambiente e à saúde humana, devido a sua capacidade de bioacumulação e alto tempo de permanência no meio. ${ }^{1}$

Os metais tóxicos são fontes potenciais de degradação ambiental, visto que eles produzem alterações na qualidade das águas e do solo. Estas alterações têm um impacto direto no equilíbrio dos ecossistemas ambientais, afetando direta ou indiretamente a saúde dos seres humanos, sendo que alguns desses metais são capazes de provocar efeitos tóxicos agudos e câncer. ${ }^{2}$ Dentre os metais tóxicos, o cromo se destaca devido à grande gama de utilização em diversos processos industriais, como na indústria do aço e de ligas, indústria de cimento, galvanoplastia, curtumes, entre outras. ${ }^{3}$

Todas as formas de cromo podem ser tóxicas em grandes concentrações, sendo a hexavalente cerca de cem vezes mais tóxica do que a trivalente. ${ }^{4} \mathrm{Na}$ forma trivalente o cromo é essencial ao metabolismo humano e sua carência causa doenças. Já na forma hexavalente é tóxico e cancerígeno. ${ }^{5}$ Os compostos de cromo hexavalente como cromatos, dicromatos e o ácido crômico são extremamente tóxicos e a ingestão de pequenas quantidades pode ser fatal. ${ }^{6}$ Os efluentes contendo cromo (VI) possuem alto poder de contaminação necessitando tratamento adequado para posterior destinação final. ${ }^{5}$

A capacidade bioacumulativa dos metais gera efeitos em longo prazo que nem sempre são previsíveis, principalmente no caso de compostos como o cromo, que não se decompõem ou que apresentam baixa degradabilidade, permanecendo ativo no meio ambiente, onde são absorvidos pelos organismos em concentrações muito maiores do que as de seu lançamento inicial. ${ }^{7} \mathrm{O}$ metal no solo pode ser absorvido por plantas que posteriormente servirão de alimento diretamente ao homem ou aos animais. ${ }^{8}$ No ambiente, o cromo atinge os lençóis freáticos com facilidade, ou mesmo reservatórios ou rios que são as fontes de abastecimento de água para a população. ${ }^{9}$

Diante da grande variedade de fontes geradoras de efluentes contendo cromo (VI), o desenvolvimento de novas tecnologias para remoção desse elemento vem sendo intensamente estudado. ${ }^{10}$ Vários métodos vêm sendo utilizados para remoção de íons metálicos de soluções, tais como precipitação química, ${ }^{11,12}$ troca iônica, ${ }^{13}$ adsorção ${ }^{14,15}$ e tratamento eletroquímico, ${ }^{16}$ mas nem sempre são eficientes e apresentam-se com elevado custo, ${ }^{17}$ principalmente quando aplicados a soluções diluídas de metais. ${ }^{18}$ Portanto, a busca de novas tecnologias de tratamento desses efluentes que apresente vantagens em relação ao custo-benefício do processo vem direcionando ao aumento no interesse sobre o processo de biossorção, ${ }^{17}$ que consiste na absorção de metais através de microrganismos ativos (biossorção ativa) ou inativos (biossorção passiva). ${ }^{18}$

A biossorção é uma alternativa eficaz aos tratamentos de efluentes convencionais já utilizados, visto que os microrganismos retêm os metais promovendo um polimento do efluente e qualificando o processo, ${ }^{19}$ tornando-se uma tecnologia promissora para tratamento de efluentes contendo metais tóxicos, ${ }^{20,21}$ de forma a garantir o adequado tratamento desse efluente e reduzir os impactos ambientais causados por este no momento do lançamento no meio ambiente.

Diferentes materiais biológicos vêm sendo empregados visando a biossorção de metais tóxicos presentes em efluentes, tais como biomassas de algas, ${ }^{22-24}$ fungos, ${ }^{25,26}$ leveduras, ${ }^{27,9}$ bactérias, ${ }^{28,29} \mathrm{e}$ macrófitas aquáticas. ${ }^{20,30}$

As algas podem ser utilizadas no tratamento de águas residuais, desintoxicação biológica e controle de metais tóxicos em águas naturais ou em águas contaminadas industrialmente, devido a sua capacidade de retenção e imobilização desses compostos, ${ }^{31}$ sendo que as algas podem acumular metais na sua parede externa por meio de mecanismos físicos, químicos e biológicos. ${ }^{32}$ Dentre os microrganismos utilizados, em diversos estudos a microalga Spirulina platensis tem apresentado capacidade de remoção de íons metálicos de soluções aquosas. ${ }^{33,34}$

Considerando que o cromo apresenta alto potencial de degradação ambiental, principalmente na sua forma hexavalente, e que a microalga Spirulina platensis tem sido utilizada no processo de biossorção de metais tóxicos, esse trabalho teve como objetivo avaliar a capacidade da biomassa inativa (biossorção passiva) dessa microalga para biossorção de cromo (VI). 
Tabela 1. Planejamento Fatorial Fracionário (PFF) ${ }^{6-2}$ IV para avaliação das variáveis que apresentam influência significativa sobre o processo de biossorção passiva pela microalga Spirulina platensis

\begin{tabular}{|c|c|c|c|c|c|c|}
\hline Exp. & $\begin{array}{c}\mathrm{X}_{1} \\
\text { ([biossorvente }]-\mathrm{g} \mathrm{L}^{-1} \text { ) }\end{array}$ & $\begin{array}{c}\mathrm{X}_{2} \\
\text { (pH inicial) }\end{array}$ & $\begin{array}{c}\mathrm{X}_{3} \\
\text { (Tempo reacional - } \mathrm{h} \text { ) }\end{array}$ & $\begin{array}{c}\mathrm{X}_{4} \\
\text { (agitação - rpm) }\end{array}$ & $\begin{array}{c}\mathrm{X}_{5} \\
\text { (Pré-tratamento) }\end{array}$ & $\begin{array}{c}\mathrm{X}_{6} \\
\text { ([inicial de cromo }]-\mathrm{mg} \mathrm{L}^{-1} \text { ) }\end{array}$ \\
\hline 1 & $-1(2,5)$ & $-1(5)$ & $-1(1)$ & $-1(100)$ & -1 (não) & $-1(50)$ \\
\hline 2 & $1(5)$ & $-1(5)$ & $-1(1)$ & $-1(100)$ & $1(\operatorname{sim})$ & $-1(50)$ \\
\hline 3 & $-1(2,5)$ & $1(9)$ & $-1(1)$ & $-1(100)$ & $1(\operatorname{sim})$ & $1(100)$ \\
\hline 4 & $1(5)$ & $1(9)$ & $-1(1)$ & $-1(100)$ & -1 (não) & $1(100)$ \\
\hline 5 & $-1(2,5)$ & $-1(5)$ & $1(3)$ & $-1(100)$ & $1(\operatorname{sim})$ & $1(100)$ \\
\hline 6 & $1(5)$ & $-1(5)$ & $1(3)$ & $-1(100)$ & -1 (não) & $1(100)$ \\
\hline 7 & $-1(2,5)$ & $1(9)$ & $1(3)$ & $-1(100)$ & -1 (não) & $-1(50)$ \\
\hline 8 & $1(5)$ & $1(9)$ & $1(3)$ & $-1(100)$ & $1(\operatorname{sim})$ & $-1(50)$ \\
\hline 9 & $-1(2,5)$ & $-1(5)$ & $-1(1)$ & $1(150)$ & -1 (não) & $1(100)$ \\
\hline 10 & $1(5)$ & $-1(5)$ & $-1(1)$ & $1(150)$ & $1(\operatorname{sim})$ & $1(100)$ \\
\hline 11 & $-1(2,5)$ & $1(9)$ & $-1(1)$ & $1(150)$ & $1(\operatorname{sim})$ & $-1(50)$ \\
\hline 12 & $1(5)$ & $1(9)$ & $-1(1)$ & $1(150)$ & -1 (não) & $-1(50)$ \\
\hline 13 & $-1(2,5)$ & $-1(5)$ & $1(3)$ & $1(150)$ & $1(\operatorname{sim})$ & $-1(50)$ \\
\hline 14 & $1(5)$ & $-1(5)$ & $1(3)$ & $1(150)$ & -1 (não) & $-1(50)$ \\
\hline 15 & $-1(2,5)$ & $1(9)$ & $1(3)$ & $1(150)$ & -1 (não) & $1(100)$ \\
\hline 16 & $1(5)$ & $1(9)$ & $1(3)$ & $1(150)$ & $1(\operatorname{sim})$ & $1(100)$ \\
\hline
\end{tabular}

\section{PARTE EXPERIMENTAL}

\section{Ensaios de biossorção passiva}

Os ensaios de biossorção passiva foram desenvolvidos com a biomassa inativa da microalga Spirulina platensis obtida a partir de cultivos realizados em planta piloto localizada na cidade de Santa Vitória do Palmar - RS, cedidas pelo Laboratório de Engenharia Bioquímica da Universidade Federal do Rio Grande.

Todos os ensaios de biossorção passiva foram realizados em duplicatas, utilizando-se erlenmeyers de $300 \mathrm{~mL}$, com volume inicial de $100 \mathrm{~mL}$ de solução de dicromato de potássio $\left(\mathrm{K}_{2} \mathrm{Cr}_{2} \mathrm{O}_{7}\right)$ contendo concentrações variadas de $50 \mathrm{mg} \mathrm{L}^{-1}$ de cromo (VI) e $100 \mathrm{mg} \mathrm{L}^{-1}$ de cromo (VI), os quais foram submetidos a agitações variáveis em agitador orbital (100 rpm e $150 \mathrm{rpm})$, com temperatura constante de $30{ }^{\circ} \mathrm{C}$, durante períodos de tempo ( $1 \mathrm{~h}$ e $3 \mathrm{~h}$ ) e concentrações de biossorvente de $2,5 \mathrm{~g} \mathrm{~L}^{-1}$ e $5,0 \mathrm{~g} \mathrm{~L}^{-1}$. O pré-tratamento da biomassa foi realizado em autoclave a $121^{\circ} \mathrm{C}$ durante $20 \mathrm{~min}$, sendo utilizados $\mathrm{pHs}$ também variáveis ( 5 e 9), conforme Planejamento Fatorial Fracionário (PFF) ${ }^{6-2}$ IV apresentado na Tabela 1.

Após o processo de biossorção, as amostras foram centrifugadas durante 15 min a $6000 \mathrm{rpm}$ e filtradas (papel filtro Whatman 40) para retirada da parte sólida e posterior determinação de cromo (VI), através do método da 1,5 - difenilcarbazida, conforme Standard methods for the examination of water and wastewater ${ }^{35}$ e realização dos cálculos dos percentuais de remoção, conforme Equação 1.

$$
\% \text { remoção }=\left(1-\frac{C_{f}}{C_{0}}\right) \times 100
$$

sendo: $C_{f}=$ concentração final $\left(\mathrm{mg} \mathrm{L}^{-1}\right) ; C_{0}=$ concentração inicial $\left(\mathrm{mg} \mathrm{L}^{-1}\right)$

A partir dos resultados do Planejamento Fatorial Fracionário $2^{6-2}$, as variáveis que apresentavam maior influência no processo de biossorção de cromo (VI) foram testadas novamente com variações em torno do nível que apresentou maior remoção do metal pela biomassa, conforme Planejamento Experimental $2^{3}$ com três pontos centrais apresentado na Tabela 2.

Tabela 2. Planejamento Experimental $2^{3}$ com três pontos centrais para avaliação da remoção de cromo (VI) a partir das variáveis que apresentaram maior influência no processo biossortivo do PFF $2_{\text {IV }}^{6-2}$

\begin{tabular}{cccc}
\hline Exp. & $\mathrm{X}_{1}$ & $\mathrm{X}_{2}$ & $\mathrm{X}_{3}$ \\
\hline 1 & -1 & -1 & -1 \\
2 & 1 & -1 & -1 \\
3 & -1 & 1 & -1 \\
4 & 1 & 1 & -1 \\
5 & -1 & -1 & 1 \\
6 & 1 & -1 & 1 \\
7 & -1 & 1 & 1 \\
8 & 1 & 1 & 1 \\
9 & 0 & 0 & 0 \\
10 & 0 & 0 & 0 \\
11 & 0 & 0 & 0 \\
\hline
\end{tabular}

$\mathrm{X}_{1}, \mathrm{X}_{2}$ e $\mathrm{X}_{3}$ : variáveis mais influentes no PFF $2^{6-2}$.

Outro aspecto que foi considerado foi a possibilidade de ocorrência de dessorção do metal ligado a biomassa em função do tempo de contato. Para verificar tal fenômeno, foi realizada a determinação da remoção de cromo VI ao longo do tempo, mantendo-se a concentração inicial de biomassa e de cromo (VI) constantes nos níveis das variáveis que apresentaram melhores resultados de remoção de cromo (VI) no Planejamento Fatorial Fracionado e variando-se os tempos de análise de cromo (VI) entre 10 min e 48 h.

\section{Análise dos resultados}

A avaliação dos efeitos estimados das variáveis sobre as variáveis resposta e coeficientes de regressão dos modelos foram gerados pelo 
Software Statistica vs. 6.0, sendo feita a análise de variância pelo módulo Experimental Design, utilizando-se nível de significância de $5 \%$. A análise de variância foi realizada para verificar a influência das variáveis independentes (entrada) sobre as variáveis dependentes (resposta) no processo de biossorção de cromo (VI) pela biomassa inativa da Spirulina platensis.

\section{Equilíbrio de adsorção}

Com o intuito de construir a curva de equilíbrio para a adsorção do cromo (VI) pela biomassa inativa da microalga foram realizados ensaios variando-se a concentração de biomassa no meio e fixando-se o pH e a concentração inicial de cromo (VI), conforme apresentado na Tabela 3. O tempo utilizado para análise da remoção de cromo (VI) foi o tempo em que se alcançou a melhor remoção de cromo (VI) no teste de biossorção. Ao final do processo foi novamente medido o $\mathrm{pH}$ dos ensaios para verificar se o mesmo permaneceu constante, permitindo a isometria do processo.

Tabela 3. Dados utilizados para construção da isoterma

\begin{tabular}{cccc}
\hline Exp. & $\begin{array}{c}{[\text { biossorvente }]} \\
\left(\mathrm{g} \mathrm{L}^{-1}\right)\end{array}$ & $(\mathrm{pH}$ inicial $)$ & $\begin{array}{c}\text { [inicial de cromo }(\mathrm{VI})] \\
\left(\mathrm{mg} \mathrm{L}^{-1}\right)\end{array}$ \\
\hline 1 & 0,25 & Fixo* & Fixa* \\
2 & 0,5 & Fixo* & Fixa* \\
3 & 0,75 & Fixo* & Fixa* \\
4 & 1,0 & Fixo* & Fixa* \\
5 & 1,5 & Fixo* & Fixa* \\
6 & 2,0 & Fixo* & Fixa* \\
7 & 2,5 & Fixo* & Fixa* \\
8 & 5,0 & Fixo* & Fixa* \\
\hline
\end{tabular}

* Melhor condição de pH inicial e de concentração inicial de cromo (VI) obtidas nos ensaios do Planejamento Experimental $2^{3}$.

Para construção da curva de equilíbrio de fases foram utilizados os resultados da concentração de equilíbrio do metal na solução (quantidade de cromo (VI) ao final do ensaio obtido a partir da curva padrão predefinida) $\left(\mathrm{C}_{\mathrm{e}} \mathrm{em} \mathrm{mg} \mathrm{L}^{-1}\right)$ e do cálculo da quantidade de metal adsorvido na biomassa seca $\left(\mathrm{q}_{\mathrm{e}} \mathrm{em} \mathrm{mg} \mathrm{g}^{-1}\right)$, calculados de acordo com a Equação 2. A temperatura do ensaio foi fixada em $30^{\circ} \mathrm{C}$.

$$
q_{e}=\left(\frac{\left(C_{o}-C_{e}\right) V}{M}\right)
$$

sendo: $C_{o}=$ concentração inicial do metal em solução $\left(\mathrm{mg} \mathrm{L}^{-1}\right) ; C_{e}=$ concentração em equilíbrio do metal em solução $\left(\mathrm{mg} \mathrm{L}^{-1}\right) ; \mathrm{V}=$ volume da solução $(\mathrm{L}) ; \mathrm{M}=$ massa de biossorvente $(\mathrm{g})$

\section{RESULTADOS E DISCUSSÃO}

\section{Influência de variáveis sobre a remoção de cromo (VI)}

Os resultados de remoção de cromo (VI) obtidos nos experimentos do Planejamento Fatorial Fracionário (PFF) $2_{\mathrm{IV}}^{6-2}$, realizado através do processo de biossorção passiva pela microalga Spirulina platensis estão representados de forma gráfica na Figura 1.

Com base nos dados apresentados na Figura 1, observou-se que as maiores remoções de cromo (VI) $(61,97 \%, 60,09 \%$ e $57,75 \%)$ foram atingidas nos experimentos 1,2 e 9 , respectivamente, demonstrando que os melhores resultados foram obtidos no menor valor de $\mathrm{pH}$ (5) e no menor tempo reacional $(1 \mathrm{~h})$.

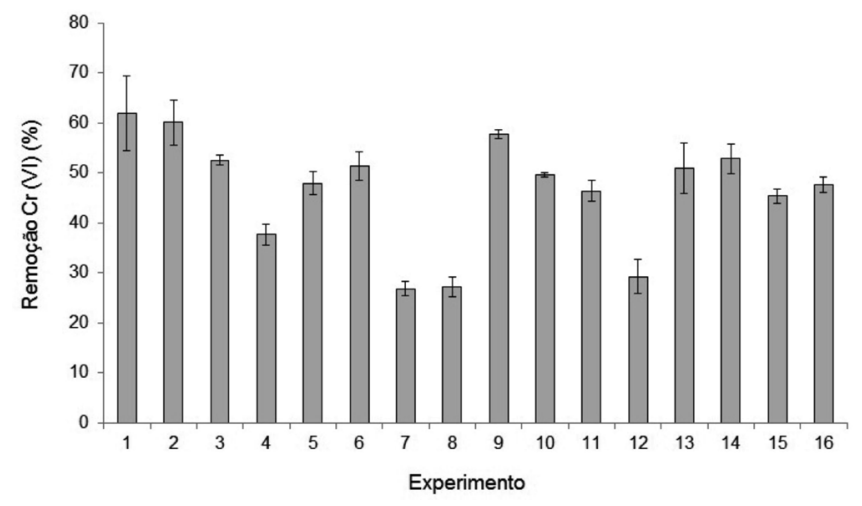

Figura 1. Remoções de cromo (VI) no processo de biossorção passiva com a microalga $\mathrm{S}$. platensis segundo os ensaios do planejamento Fatorial Fracionário $(P F F) 2^{6-2}$ e respectivos desvios padrões

Gokhale et al. ${ }^{36}$ realizaram ensaios de biossorção de cromo (VI) em colunas empacotadas utilizando a biomassa seca da Spirulina platensis como material adsorvente, atingindo, em condições experimentais otimizadas, com pH 1,5, agitação de $180 \mathrm{rpm}$ e temperatura de $30^{\circ} \mathrm{C}$, a remoção de até $99 \%$ do metal para uma solução contendo $100 \mathrm{mg} \mathrm{L}^{-1}$ de cromo (VI). Aneja et al. ${ }^{37}$ avaliaram a biossorção de $\mathrm{Pb}^{+2} \mathrm{e} \mathrm{Zn}^{+2}$ de uma solução aquosa contendo diferentes concentrações desses metais, através da biomassa inativa da Spirulina sp., atingindo índices de biossorção de $82 \%$ para $\mathrm{Pb}^{+2}$ e de $90 \%$ para $\mathrm{Zn}^{+2}$ para um tempo de contato de $15 \mathrm{~min}$, porém, para o $\mathrm{pH}$, os melhores resultados foram obtidos em valores elevados, com os melhores resultados de remoção de $\mathrm{Pb}^{+2}$ obtidos nos pHs 4 e 10 , enquanto para o $\mathrm{Zn}^{+2}$ as melhores remoções foram nos pHs 8 e 10 .

A Tabela 4 apresenta os efeitos estimados das variáveis estudadas no PFF sobre o percentual de remoção de Cromo (VI) do meio líquido $(\mathrm{p}<0,05)$.

Tabela 4. Efeitos estimados das variáveis do PFF $2^{6-2}$ IV sobre a remoção de cromo (VI) com a biomassa de Spirulina platensis inativa

\begin{tabular}{lcccc}
\hline Fonte de variação & $\begin{array}{c}\text { Efeitos } \\
\text { estimados }\end{array}$ & $\begin{array}{c}\text { Erro } \\
\text { padrão }\end{array}$ & $\mathrm{t}(25)$ & $\mathrm{p}$ \\
\hline Média & 46,5951 & 1,317058 & 35,37819 & 0,000000 \\
(1) [biossorvente] & $-4,2710$ & 2,634117 & $-1,62141$ & 0,117477 \\
(2) $\mathrm{pH}$ & $-14,9152$ & 2,634117 & $-5,66233$ & 0,000007 \\
(3) Tempo reacional & $-5,6566$ & 2,634117 & $-2,14744$ & 0,041645 \\
(4) Agitação & 1,7797 & 2,634117 & 0,67565 & 0,505467 \\
(5) Pré-tratamento & 2,4269 & 2,634117 & 0,92132 & 0,365687 \\
(6) [Cr(VI)] inicial & 4,3062 & 2,634117 & 1,63479 & 0,114624 \\
\hline
\end{tabular}

De acordo com a Tabela 4, observa-se que para a variável $\mathrm{pH}$ o efeito estimado foi negativo (-14,9152), indicando que melhores resultados de remoção de cromo (VI) foram obtidos quando trabalhou-se no menor nível da variável $(\mathrm{pH}=5)$. Segundo Cossich et al. ${ }^{38}$ o cromo hexavalente pode apresentar-se como um cromato na forma aniônica $\left(\mathrm{CrO}_{4}{ }^{2-}\right)$. Para Bayer, ${ }^{39}$ em solução aquosa, o cromo pode apresentar-se na forma aniônica com os ânions cromato $\left(\mathrm{CrO}_{4}\right)^{2-}$ e dicromato $\left(\mathrm{Cr}_{2} \mathrm{O}_{7}\right)^{2-}$, no qual o cromo tem um estado de oxidação +6 . Segundo Kuyucak e Volesky, ${ }^{40}$ com a diminuição do $\mathrm{pH}$, grupos funcionais tais como amina e carbonila fornecem superfícies celulares carregadas positivamente, favorecendo a biossorção de metais presentes como espécies aniônicas. Para Blázquez et al. ${ }^{41}$ a queda na capacidade de adsorção em pH acima de 5,0 se deve à formação de complexos hidratados dos metais e alterações eletrostáticas na superfície da alga. 
Alguns autores realizaram testes de biossorção de cromo (VI) em função do $\mathrm{pH}$, concluindo que os melhores resultados foram obtidos com $\mathrm{pH}$ na faixa de 3 a 5, ${ }^{42-44}$ confirmando os melhores resultados obtidos nesse trabalho, os quais foram obtidos com baixo valor de $\mathrm{pH}$.

Em relação ao tempo reacional (TR), observou-se que melhores resultados foram obtidos em tempos inferiores $(\mathrm{TR}=1 \mathrm{~h}$ ) (efeito de $-5,6566)$, demonstrando que o aumento no tempo reacional reduziu os percentuais de remoção de cromo (VI). Esse fato levantou a suspeita de que poderia estar havendo a dessorção do metal aderido à superfície do biossorvente no início do processo.

Segundo Kotrba et $a l .{ }^{45}$ a adsorção pode ser física (ligações fracas) e/ou químicas (ligações fortes que envolvem o íon metálico por complexação e/ou quelação). O fato da obtenção de melhores resultados em menor tempo reacional pode significar que a ligação física predominou diante da ligação química, gerando ligações fracas entre o biossorvente e o metal, acarretando, quando em maior tempo reacional, a dessorção dos íons metálicos. ${ }^{45}$

A fim de explorar melhor o efeito do tempo reacional sobre a remoção de cromo (VI), foi realizado um ensaio para verificar se ao longo do tempo poderia haver queda significativa no percentual de remoção de cromo (VI), indicando uma possível dessorção do metal. Para tanto, foi realizado um ensaio com concentração de biossorvente de 2,5 g L-1 , concentração inicial de cromo (VI) de $100 \mathrm{mg} \mathrm{L}^{-1}$ e pH 5, avaliando-se a remoção de cromo (VI) ao longo de $48 \mathrm{~h}$. Os resultados obtidos estão demonstrado na Figura 2.

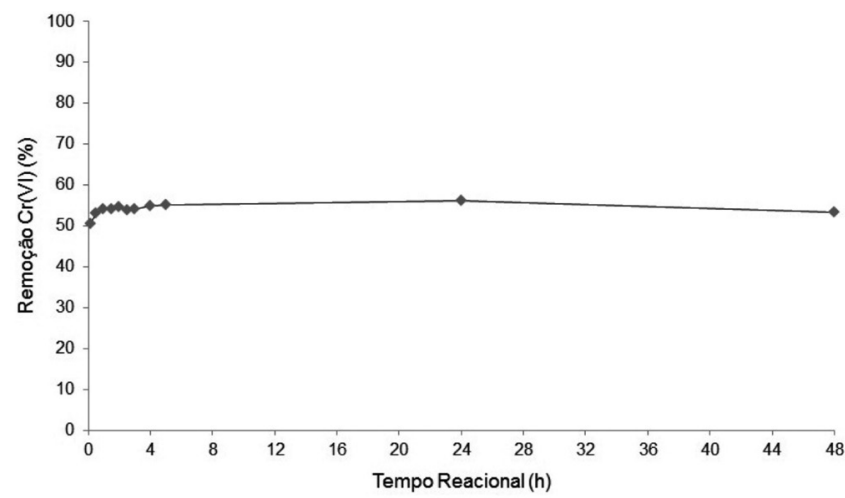

Figura 2. Remoção de cromo (VI) no efluente em função do tempo de contato com a biomassa inativa da S. platensis

Observa-se na Figura 2 que o maior percentual de biossorção de cromo (VI) foi obtido no início do ensaio (remoção de 50,43\% no tempo de $10 \mathrm{~min}$ ), configurando a predominância de ligação física entre o metal e a biomassa (ligação rápida) no início do processo. ${ }^{45}$ Ao longo do tempo o percentual de biossorção foi elevando-se gradativamente, atingindo $56,13 \%$ de remoção de cromo (VI) no tempo de $24 \mathrm{~h}$, indicando que, além da ligação física inicial, pode ter ocorrido a ligação química entre o metal e o biossorvente, gerando elos fortes e reduzindo a probabilidade de ocorrência da dessorção do metal aderido a biomassa. ${ }^{45}$ Ao longo do experimento houve leve variação na remoção de cromo, obtendo-se maior percentual de remoção de cromo (VI) no tempo de $24 \mathrm{~h}(56,13 \%)$ decaindo ao final do ensaio (48 h) para 52,74\% de remoção de cromo (VI). Essa variação observada durante o ensaio é muito pequena para configurar a dessorção do metal, sendo, portanto, descartada essa hipótese. Desta forma, foi fixado o tempo reacional em $1 \mathrm{~h}$ para desenvolvimento dos demais ensaios como forma de reduzir custos com a operação do processo.

$\mathrm{Na}$ sequência, foram desenvolvidos novos ensaios variando-se as concentrações de biomassa, pH e concentração de cromo (VI) adicionado em torno dos níveis das variáveis que apresentaram maiores efeitos no processo de biossorção do Planejamento Fatorial
Fracionário $2^{6-2}$ IV (Tabela 1), estando os resultados apresentados na Tabela 5. Os maiores percentuais de remoção de cromo (VI) foram obtidos na maior concentração de biomassa $\left(3,5 \mathrm{~g} \mathrm{~L}^{-1}\right)$ e de cromo (VI) inicial (150 $\mathrm{mg} \mathrm{L}^{-1}$ ) (Exp. 6 e Exp. 8), com remoções de 61,94\% e 58,41\%, respectivamente, nos menores valores de $\mathrm{pH}$ (Exp. 6, pH=3, $61,94 \%$ de remoção de cromo(VI)).

Tabela 5. Variáveis utilizadas e resultados obtidos conforme Planejamento Experimental $2^{3}$ a partir das variáveis mais influentes do PFF $2^{6-2}{ }_{\mathrm{IV}}$

\begin{tabular}{cccccc}
\hline Exp. & $\begin{array}{c}\mathrm{X}_{1}[\mathrm{bios}] \\
\left(\mathrm{g} \mathrm{L}^{-1}\right)\end{array}$ & $\mathrm{X}_{2} \mathrm{pH}$ & $\begin{array}{c}\mathrm{X}_{3}[\mathrm{Cr}(\mathrm{VI}) \\
\text { inicial }] \\
\left(\mathrm{mg} \mathrm{L}^{-1}\right)\end{array}$ & $\begin{array}{c}\text { \% Remoção } \\
\text { de Cr (VI)* }\end{array}$ & $\begin{array}{c}\text { Coeficiente } \\
\text { de variação } \\
(\%)\end{array}$ \\
\hline 1 & $-1(1,5)$ & $-1(3)$ & $-1(50)$ & $54,04 \pm 1,1$ & 2,10 \\
2 & $1(3,5)$ & $-1(3)$ & $-1(50)$ & $55,58 \pm 0,3$ & 0,63 \\
3 & $-1(1,5)$ & $1(5)$ & $-1(50)$ & $52,50 \pm 1,0$ & 1,99 \\
4 & $1(3,5)$ & $1(5)$ & $-1(50)$ & $51,88 \pm 0,5$ & 1,01 \\
5 & $-1(1,5)$ & $-1(3)$ & $1(150)$ & $53,46 \pm 0,4$ & 0,67 \\
6 & $1(3,5)$ & $-1(3)$ & $1(150)$ & $61,94 \pm 0,5$ & 0,84 \\
7 & $-1(1,5)$ & $1(5)$ & $1(150)$ & $51,47 \pm 1,5$ & 2,78 \\
8 & $1(3,5)$ & $1(5)$ & $1(150)$ & $58,41 \pm 0,1$ & 0,20 \\
9 & $0(2,5)$ & $0(4)$ & $0(100)$ & $53,91 \pm 0,7$ & 1,21 \\
10 & $0(2,5)$ & $0(4)$ & $0(100)$ & $53,42 \pm 0,5$ & 0,90 \\
11 & $0(2,5)$ & $0(4)$ & $0(100)$ & $52,37 \pm 0,8$ & 1,58 \\
\hline
\end{tabular}

* Resultados de média \pm desvio padrão. $\mathrm{X}_{1}=$ quantidade de biossorvente $\left(\mathrm{g} \mathrm{L}^{-1}\right)$; $\mathrm{X}_{2}=\mathrm{pH}$ inicial; $\mathrm{X}_{3}=$ concentração inicial de $\mathrm{Cr}(\mathrm{VI})\left(\mathrm{mg} \mathrm{L}^{-1}\right)$.

No experimento 7, com menor concentração de biomassa e maior concentração de cromo (VI) inicial, alcançou-se o menor índice de remoção de cromo (VI), de 51,47\%, indicando que a alta concentração de cromo (VI) em baixos níveis de biossorvente pode comprometer o processo biossortivo, reduzindo o potencial de captação do metal pela biomassa, podendo haver a saturação da capacidade do biossorvente em função da saturação dos sítios ativos existentes na parede celular do mesmo.

A saturação da capacidade biossortiva da biomassa representa uma desvantagem para o processo de biossorção passiva ${ }^{46} \mathrm{e} o$ incremento demasiado de biomassa no processo pode gerar uma quantidade grande de biomassa contaminada, a qual necessita de tratamento e disposição final adequados para que não gere impactos negativos ao meio ambiente, comprometendo assim a relação custo-benefício do processo. ${ }^{47,48}$

Nos demais experimentos, os índices de remoção apresentaram a mesma tendência dos experimentos 6 e 8, obtendo-se melhores resultados quando a concentração de biossorvente foi superior $\left(3,5 \mathrm{~g} \mathrm{~L}^{-1}\right)$ e o valor de $\mathrm{pH}$ inferior $(\mathrm{pH}=3)$, porém, todos os experimentos apresentaram percentuais semelhantes de remoção de cromo (VI), variando de $51,47 \%$ (Exp. 7) a 61,94\% (Exp. 6).

A análise de variância dos resultados do Planejamento Fatorial Completo $2^{3}$ (Tabela 6) confirma as hipóteses levantadas anteriormente, demonstrando que os níveis superiores das variáveis $\mathrm{X}_{1}$ e $\mathrm{X}_{3}$ (concentração de biossorvente e concentração inicial de cromo (VI)) resultam em maior biossorção de cromo VI (efeitos positivos de 2,6 e 4,8 significativos estatisticamente, $\mathrm{p}=0,011$ e $\mathrm{p}=0,002$, respectivamente). A variável $\mathrm{X}_{2}(\mathrm{pH})$ também apresentou-se significativa $(\mathrm{p}=0,010)$, porém, seu efeito foi negativo $(-2,71)$ indicando que os melhores resultados foram obtidos no menor nível estudado dessa variável $(\mathrm{pH}=3)$. A interação $\mathrm{X}_{1} \cdot \mathrm{X}_{3}$ foi significativa $(\mathrm{p}=0,020)$ apresentando efeito positivo $(2,146)$, indicando que, os maiores índices das variáveis $\mathrm{X}_{1}$ e $\mathrm{X}_{3}$ (concentração de biossorvente e concentração 
inicial de cromo (VI)), quando analisados de maneira integrada, apresentam melhores resultados.

Tabela 6. Efeitos estimados das variáveis utilizadas no Planejamento Experimental $2^{3}$ no processo de biossorção de cromo (VI) com a biomassa inativa de S. platensis

\begin{tabular}{lccc}
\hline Fonte de variação & Efeitos estimados & Erro padrão & $\mathrm{p}$ \\
\hline Média & 55,92124 & 0,237885 & 0,000000 \\
$\left(\mathrm{X}_{1}\right)$ [biossorvente] & 2,60858 & 0,475771 & 0,011933 \\
$\left(\mathrm{X}_{2}\right) \mathrm{pH}$ & $-2,71128$ & 0,475771 & 0,010715 \\
$\left(\mathrm{X}_{3}\right)[\mathrm{Cr}(\mathrm{VI})]$ inicial & 4,84223 & 0,475771 & 0,002021 \\
$\mathrm{X}_{1} \cdot \mathrm{X}_{2}$ & $-1,44807$ & 0,475771 & 0,065707 \\
$\mathrm{X}_{1} \cdot \mathrm{X}_{3}$ & 2,14643 & 0,475771 & 0,020351 \\
$\mathrm{X}_{2} \cdot \mathrm{X}_{3}$ & $-0,09243$ & 0,475771 & 0,858372 \\
\hline
\end{tabular}

A Tabela 7 apresenta as variáveis utilizadas e os resultados obtidos no estudo da curva de equilíbrio de adsorção e a Figura 3 demonstra esses resultados de forma gráfica.

Nota-se na Figura 3 que a curva de equilíbrio apresentou-se desfavorável de acordo com Devault (1943) citado por Vilar, ${ }^{3}$ pois sua forma apresenta uma tendência côncava, pressupondo-se que a adsorção é desfavorável, gerando uma frente dispersiva e comprometendo a eficiência do processo. Entretanto, o processo apresenta semelhança com a isoterma do Tipo $\mathrm{S}$ (1 ou 2) de acordo com a classificação das isotermas segundo Giles. ${ }^{49}$ Para Manosalva, ${ }^{50}$

Tabela 7. Variáveis utilizadas para construção da curva de equilíbrio e resultados de biossorção de cromo (VI) com a biomassa inativa de S. platensis

\begin{tabular}{ccccccc}
\hline Exp. & $\begin{array}{c}{[\mathrm{bios}]} \\
\left(\mathrm{g} \mathrm{L}^{-1}\right)\end{array}$ & $\begin{array}{c}\mathrm{pH} \\
\text { inicial }\end{array}$ & $\begin{array}{c}{[\mathrm{Cr}(\mathrm{VI})} \\
\text { inicial }] \\
\left(\mathrm{mg} \mathrm{L}^{-1}\right)\end{array}$ & $\begin{array}{c}\mathrm{Ce} \\
\left(\mathrm{mg} \mathrm{L}^{-1}\right)\end{array}$ & $\begin{array}{c}\mathrm{Ceq} \\
\left(\mathrm{mg} \mathrm{g}^{-1}\right)\end{array}$ & $\begin{array}{c}\mathrm{pH} \\
\text { final }\end{array}$ \\
\hline 1 & 0,25 & 3 & 100 & 74,90 & 100,39 & 3,17 \\
2 & 0,5 & 3 & 100 & 56,85 & 86,30 & 3,13 \\
3 & 0,75 & 3 & 100 & 50,75 & 65,67 & 3,35 \\
4 & 1,0 & 3 & 100 & 49,05 & 50,95 & 3,50 \\
5 & 1,5 & 3 & 100 & 41,69 & 38,87 & 3,86 \\
6 & 2,0 & 3 & 100 & 40,00 & 30,00 & 3,97 \\
7 & 2,5 & 3 & 100 & 35,50 & 25,80 & 4,10 \\
8 & 5,0 & 3 & 100 & 27,18 & 14,56 & 4,25 \\
\hline
\end{tabular}

$\mathrm{Ce}=$ concentração em equilíbrio do metal em solução $\left(\mathrm{mg} \mathrm{L}^{-1}\right)$, Ceq = quantidade de metal adsorvido na biomassa $\left(\mathrm{mg} \mathrm{g}^{-1}\right)$.

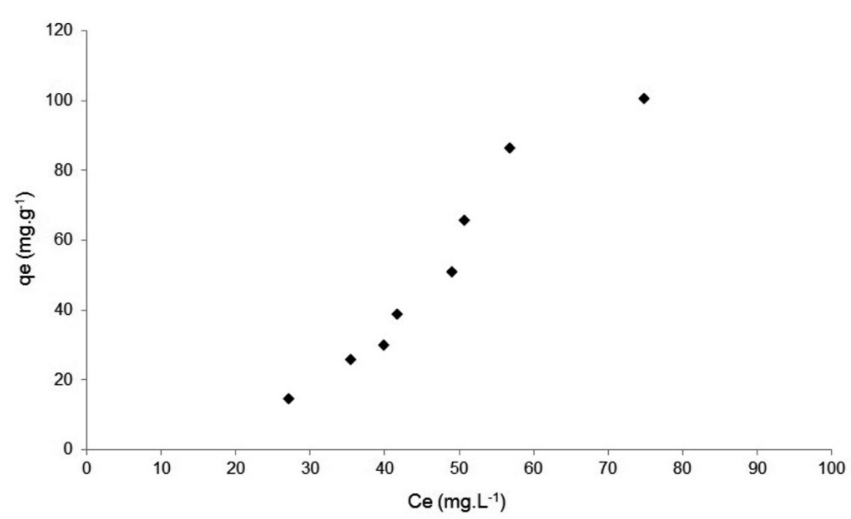

Figura 3. Resultados obtidos na construção da curva de equilíbrio de adsorção de cromo (VI) pela biomassa inativa da S. platensis esse tipo de isoterma possui um ponto de inflexão, gerando uma curva em forma sigmoidal, sugerindo que a adsorção de metais na superfície do adsorvente é favorecida devido à forte interação com outras moléculas adsorvidas.

Porém, ao analisar-se a Tabela 7, percebe-se que o $\mathrm{pH}$ sofreu diferentes alterações em relação ao valor inicial, uma vez que todos os experimentos foram iniciados com $\mathrm{pH}$ constante $(\mathrm{pH}=3$ ) e ao final o pH apresentou variações (3,17 - exp. 1 até 4,25 - exp. 8). Quanto maior a concentração de biossorvente mais alterado apresentou-se o $\mathrm{pH}$, atingindo no experimento 8 (maior concentração de biossorvente $-5,0 \mathrm{~g} \mathrm{~L}^{-1}$ ) o valor de 4,25.

Em condições ácidas, os átomos de hidrogênio $\left(\mathrm{H}^{+}\right)$podem protonar os grupos amina $\left(\mathrm{NH}_{2}\right)$ da biomassa, ocasionando alterações no $\mathrm{pH}$ da solução. ${ }^{51} \mathrm{O}$ aumento do $\mathrm{pH}$ da solução pode tornar a parede celular da biomassa menos positiva, ocasionando a repulsão entre os aníons (no caso cromato e dicromato) e a parede do adsorvente. ${ }^{52}$

Conforme visto anteriormente, a elevação do $\mathrm{pH}$ reduz a capacidade de retenção de cromo (VI) pela biomassa morta da S. platensis, uma vez que, em solução aquosa, o cromo pode se apresentar na forma aniônica como ânions cromato $\left(\mathrm{CrO}_{4}\right)^{2-}$ e dicromato $\left(\mathrm{Cr}_{2} \mathrm{O}_{7}\right)^{2-}$, no qual o cromo tem um estado de oxidação +6 . $^{38,39}$

A variação do $\mathrm{pH}$ em relação ao valor inicial pode ter comprometido a isometria do processo e diminuído a eficiência na retenção do metal pela biomassa, influenciando na eficácia do processo de biossorção passiva de cromo (VI) pela S. platensis.

Em termos de adsorção do metal pela biomassa $\left(q_{\mathrm{e}}\right)$, observa-se na Tabela 7 que a microalga apresentou potencial de adsorver cromo (VI), variando de 14,56 $\mathrm{mg} \mathrm{g}^{-1}$ (Exp. 8) a 100,39 $\mathrm{mg} \mathrm{g}^{-1}$ (Exp. 1). Nota-se que quanto maior a quantidade de biomassa menor foi a $\mathrm{q}_{\mathrm{e}}$ (Exp. $8-5 \mathrm{~g} \mathrm{~L}^{-1}$ de biomassa $-\mathrm{q}_{\mathrm{e}}=14,56 \mathrm{mg} \mathrm{g}^{-1}$ ), sendo perfeitamente justificável uma vez que a $\mathrm{q}_{\mathrm{e}}$ é inversamente proporcional a quantidade de biossorvente existente no meio (vide Equação 2).

Em comparação com resultados obtidos por outros autores, Doshi e colaboradores ${ }^{34}$ avaliaram o potencial da Spirulina inativa e ativa para biossorção dos metais $\mathrm{Cr}^{3+}, \mathrm{Ni}^{2+}, \mathrm{Cu}^{2+} \mathrm{e} \mathrm{Cr}^{6+}$ (na forma de $\mathrm{Cr}_{2} \mathrm{O}_{7}{ }^{2-}$ ). Os autores utilizaram diferentes concentrações de metais $(0,05 \mathrm{~g} / 25 \mathrm{ml} \mathrm{a} 0,5 \mathrm{~g} / 25 \mathrm{ml})$ com concentração constante de biomassa $(0,3 \mathrm{~g} / 25 \mathrm{ml})$. Com a biomassa inativa obtiveram resultados de $130 \mathrm{mg} \mathrm{g}^{-1}, 100 \mathrm{mg} \mathrm{g}^{-1}, 167 \mathrm{mg} \mathrm{g}^{-1}, 515 \mathrm{mg} \mathrm{g}^{-1}$ para $\mathrm{Cu}^{2+}, \mathrm{Cr}^{6+}, \mathrm{Cr}^{3+}, \mathrm{Ni}^{2+}$, respectivamente, enquanto que com a biomassa ativa atingiram remoções de $389 \mathrm{mg} \mathrm{g}^{-1}, 333 \mathrm{mg} \mathrm{g}^{-1}, 304 \mathrm{mg} \mathrm{g}^{-1}$, $1.378 \mathrm{mg} \mathrm{g}^{-1}$ para $\mathrm{Cu}^{2+}, \mathrm{Cr}^{6+}, \mathrm{Cr}^{3+}, \mathrm{Ni}^{2+}$, respectivamente. Ao final do estudo, os autores concluíram que a microalga apresenta-se com potencial para biossorção de metais. Esse estudo demonstra uma semelhança com o atual trabalho na eficiência do processo de biossorção do metal cromo (VI) pela biomassa inativa da Spirulina, uma vez que os autores mencionados atingiram capacidade de biossorção de $100 \mathrm{mg} \mathrm{g}^{-1}$, valor esse muito próximo ao encontrado no atual trabalho, o qual atingiu capacidade de biossorção de 100,39 $\mathrm{mg} \mathrm{g}^{-1}$ (Exp. 1 - Tabela 7).

Gokhale et al..$^{36}$ realizaram um estudo de biossorção de cromo (VI) pela biomassa de Spirulina platensis imobilizada em cápsulas de alginato de cálcio. Na ocasião, os autores utilizaram solução metálica com concentração inicial de cromo (VI) variável de $100 \mathrm{mg} \mathrm{L}^{-1}$, $150 \mathrm{mg} \mathrm{L}^{-1}, 200 \mathrm{mg} \mathrm{L}^{-1}$ e concentrações de biomassa variáveis de acordo com a altura do leito da coluna $(15 \mathrm{~cm}, 22 \mathrm{~cm}$ e $28 \mathrm{~cm})$, alcançando resultados de até $99 \%$ de remoção de cromo (VI) com maior altura do leito e concentração inicial do metal de $100 \mathrm{mg} \mathrm{L}^{-1}$. Doshi et al..$^{53}$ compararam o potencial das algas verdes Cladophora sp. e Spirulina sp. para biossorção dos metais $\mathrm{Cr}^{3+}, \mathrm{Ni}^{2+}, \mathrm{Cu}^{2+} \mathrm{e}$ $\mathrm{Cr}^{6+}$ (na forma de $\mathrm{Cr}_{2} \mathrm{O}_{7}^{2-}$ ) com diferentes concentrações metálicas $(0,1 \mathrm{~g} / 25 \mathrm{~mL}$ a $0,5 \mathrm{~g} / 25 \mathrm{~mL})$ e com concentração constante de biomassa $(0,3 \mathrm{~g} / 25 \mathrm{ml})$. Para a Cladophora $\mathrm{sp}$. obtiveram remoções 
de $\mathrm{Cu}^{2+}, \mathrm{Ni}^{2+}, \mathrm{Cr}^{3+}$ e $\mathrm{Cr}^{6+}$ de $819 \mathrm{mg} \mathrm{g}^{-1}, 504 \mathrm{mg} \mathrm{g}^{-1}, 347 \mathrm{mg} \mathrm{g}^{-1} \mathrm{e}$ $168 \mathrm{mg} \mathrm{g}^{-1}$, respectivamente, enquanto que com a Spirulina sp. as remoções foram de $576 \mathrm{mg} \mathrm{g}^{-1}, 1.108 \mathrm{mg} \mathrm{g}^{-1}, 306 \mathrm{mg} \mathrm{g}^{-1}$ e $202 \mathrm{mg} \mathrm{g}^{-1}$ para $\mathrm{Cu}^{2+}, \mathrm{Ni}^{2+}, \mathrm{Cr}^{3+}$ e $\mathrm{Cr}^{6+}$, respectivamente. Com esses resultados, os autores concluíram que fica evidente o potencial de ambas as algas para remoção de metais tóxicos.

Diversos micro-organismos têm sido utilizados para captação de uma gama diversificada de metais tóxicos. Mane et al..$^{32}$ estudaram o potencial das algas Spirogyra sp e Nostoc commune na remoção de

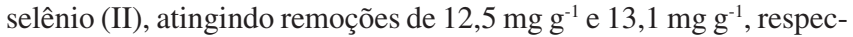
tivamente, sendo os melhores resultados obtidos quando a biomassa de ambas as algas foram submetidas ao pré-tratamento físico de secagem em forno a $60{ }^{\circ} \mathrm{C}$ durante $12 \mathrm{~h}$. Monteiro et al. ${ }^{24}$ estudaram a capacidade da microalga Scenedesmus obliquus para biossorção de zinco. A maior remoção de zinco ocorreu quando utilizada maior concentração de metal (75 mg de $\mathrm{Zn} \mathrm{L}^{-1}$ ) alcançando $836 \mathrm{mg}$ de $\mathrm{Zn}$ adsorvido por grama de biomassa, demonstrando elevado potencial dessa microalga para captação de metais.

Ferreira et al. ${ }^{27}$ avaliaram o potencial da levedura Saccharomyces cerevisiae para captação do metal $\mathrm{Pb}^{2+}$. Para o ajuste dos dados experimentais usando o modelo de Langmuir com regressão não linear, a capacidade máxima dos íons de chumbo adsorvidos pela Saccharomyces cerevisiae foi de $1,48 \mathrm{~g} \mathrm{~g}^{-1}$, enquanto com ajuste dos dados com regressão linear obteve-se capacidade máxima de 1,2 $\mathrm{g} \mathrm{g}^{-1}$, sendo que maiores quantidades de biossorção, por unidade de biomassa, foram obtidas com concentrações menores de biomassa. Khani et al. ${ }^{26}$ utilizaram a biomassa do fungo Aspergillus terreus tratada com $\mathrm{NaOH}$ para biossorção de estrôncio $(\mathrm{Sr})$ em diferentes valores de $\mathrm{pH}$, temperatura e concentração de metal. O melhor resultado foi obtido com concentração metálica de $876 \mathrm{mg} \mathrm{L} \mathrm{de}$ Sr, pH 9 e temperatura de $15{ }^{\circ} \mathrm{C}$, atingindo adsorção de $308 \mathrm{mg}$ de Sr por g de biomassa.

\section{CONCLUSÕES}

Os ensaios de biossorção passiva apresentaram resultados de remoção de cromo (VI) de até $61,97 \%$, sendo que a variável que apresentou maior influência no processo foi o $\mathrm{pH}$, com melhores resultados de biossorção de cromo (VI) obtidos em $\mathrm{pH}$ 3. O processo atingiu altos índices de remoção de cromo (VI) nos primeiros 10 min de reação, indicando o grande potencial da biomassa microalgal para uso em processos de biossorção. Considerando que a biomassa possa ser obtida a partir do crescimento em meios líquidos contendo efluentes, considera-se que a microalga apresenta importância para o uso em aplicações ambientais. A capacidade adsortiva atingida pela biomassa inativa da Spirulina platensis foi de 100,39 mg de cromo (VI) por g de biomassa.

\section{REFERÊNCIAS}

1. Jimenez, R. S.; Dal Bosco, S. M.; Carvalho, W. A.; Quím. Nova 2004, $27,734$.

2. Batista, F. G. A.; Freire, J. A.; Engenharia Ambiental: Pesquisa e Tecnologia 2010, 7, 166.

3. Vilar, V. J. P.; Tese de Doutorado, Universidade do Porto, Portugal, 2006.

4. Ribeiro, I. C. M.; Rosolem, J. C.; Grubhofer, N. J.; Andrades, S. A.; Seminário Metais Pesados: O Cromo e o Meio Ambiente. Centro Universitário Franciscano, Curitiba, Brasil, 2009.

5. Muniz, K. P. M. S.; Castilhos, Z. C.; Egler, S. G.; Resumos da XIV Jornada de Iniciação Científica. Centro de Tecnologia Mineral (CETEM), Rio de Janeiro, Brasil, 2006.

6. Korzenowski, C.; Tese de Doutorado, Universidade Federal do Rio Grande do Sul, Porto Alegre, Brasil, 2007.
7. Freitas, T. C. M.; Melnikov, P.; Eng. Sanit. Ambient. 2006, 11, 305.

8. Merlino, L. C. S.; Melo, W. J.; Macedo, F. G.; Guedes, A. C. T. P.; Ribeiro, M. H.; Melo, V. P.; Melo, G. M. P.; Rev. Bras.Cienc. Solo 2010, 34, 2031.

9. Canuto, M. F. C. S.; Ferreira, J. M.; Da Silva, F. L. H.; Alsina, O. L. S.; Oliveira, L. S. C.; Cavalcante, E. B.; Gomes, W. C.; Medeiros, M. R.; Revista Eletrônica de Materiais e Processos 2007, 2, 29.

10. http://www.ufscar.br/ enemp2002/pi32.pdf, acessada em Dezembro 2012.

11. Janegitz, B. C.; Lourenção, B. C.; Lupetti, K. O.; Fatibello-Filho, O.; Quím. Nova 2007, 30, 879.

12. Matlock, M. M.; Howerton, B. S.; Atwood, D. A.; Water Res., 2002, 36, 4757.

13. Dabrowski, A.; Hubicki, Z.; Podkoscielny, P.; Robens, E.; Chemosphere 2004, 56, 91 .

14. Detcheva, A.K., Vassileva, P.S., Georgieva, R.H., Voykova, D.K., Gerganova, T.I.; Ivanova, Y.Y.; Cent. Eur. J. Chem. 2011, 9, 932.

15. Mohammad, M.; Maitra, S.; Ahmad, N.; Bustam, A.; Sen, T. K.; Dutta, B. K.; J. Hazard. Mater. 2010, 179, 363.

16. Hunsom, M.; Pruksathorn, K.; Damronglerd, S.; Vergnes, H.; Duverneuil, P.; Water Res. 2005, 39, 610.

17. Das, N.; Vimala, R.; Karthika, P.; Indian J. Biotechnol. 2008, 7, 159.

18. Quintelas, C.; Fernandes, B.; Castro, J.; Figueiredo, H.; Tavares, T.; J. Hazard. Mater. 2008, 153, 799.

19. Pietrobelli, J. M. T. A.; Módenes, A. N.; Suzaki, P. Y. R.; Alflen, V. L.; Colombo, A.; Resumos do $1^{\circ}$ Simpósio de Inovação Tecnológica, Universidade Estadual do Oeste do Paraná, Cascavel, Brasil, 2008.

20. Módenes, A. N.; Pietrobelli, J. M. T. A.; Quiñones, F. R. E.; Suzaki, P. Y. R.; Alflen, V. L.; Klen, M. R. S. F.; Eng. Sanit. Ambient. 2009, 14, 465.

21. Kratochvil, D.; Volesky, B.; Trends Biotechnol. 1998, 16, 291.

22. Saravanan, A.; Brindha, V.; Manimekalai, R.; Krishnan, S.; Indian J. Sci. Technol. 2009, 2, 53.

23. Cruz, C.C.V.; Da Costa, A. C. A.; Henriques, C. A.; Luna, A. S.; Bioresour. Technol. 2004, 91, 249.

24. Monteiro, C. M.; Castro, P. M. L.; Malcat, F. X.; Environ. Chem. Lett. 2011, 9, 169.

25. Akar, T.; Tunali, S.; Bioresour. Technol. 2006, 97, 1780.

26. Khani, M. H.; Pahlavanzadeh, H.; Alizadeh, K.; Environ. Sci. Pollut. Res. Int. (2012), doi: 10.1007/s11356-012-0753-z.

27. Ferreira, J. M.; Silva, F. L. H.; Alsina, O. L. S.; Oliveira, L. S. C.; Cavalcanti, E. B.; Gomes, W. C.; Quím. Nova. 2007, 30, 1188.

28. Wierzba, S.; Proceedings of ECOpole 2010, 4, 85.

29. Chen, X.; Hu, S.; Shen, C.; Dou, C.; Shi, J.; Chen, Y.; Bioresour. Technol. 2009, 100, 330.

30. Lima, L. K. S.; Kleinübing, S. J.; E. A.; Da Silva, M. G. C.; Chem. Eng. Trans. 2011, 25, 303.

31. Vilar, V. J. P.; Botelho, C. M. S.; Boaventura, R. A. R. Em Security of Industrial Water Supply and Management, Nato Science for Peace and Security Series C: Environmental Security; Atimtay, A. T.; Sikdar, S.T., eds.; Springer: Netherlands, 2011, cap 11, p. 159.

32. Mane, P. C.; Bhosle, A. B.; Jangam, C. M.; Vishwakarma, C. V.; Advances in Applied Science Research 2011, 2, 202.

33. Arunakumara, K. K. I. U.; Xuecheng, Z.; Tropical Agricultural Research \& Extension 2007, 10, 47.

34. Doshi, H.; Ray, A.; Kothari, I. L.; Biotechnol. Bioeng. 2007, 96, 1051.

35. APHA - American Public Health Association. Standard methods for the examination of water and wastewater, $21^{\mathrm{a}}$ ed., Washington, 2005.

36. Gokhale, S. V.; Jyoti, K. K.; Lele, S. S.; J. Hazard. Mater. 2009, 170, 735.

37. Aneja, R. K.; Chaudhary, G.; Ahluwalia, S. S.; Goyal, D.; Indian J. Microbiol. 2010, 50, 438.

38. http://www.icp.csic.es/cyted/Monografias/MonografiasTeneria/capitulovii.htm, acessada em Dezembro 2012. 
39. Bayer, V.; Dissertação de Mestrado, Universidade Federal de Minas Gerais, Belo Horizonte, Brasil, 2005.

40. Kuyucak, N.; Volesky, B.; Biotechnol. Lett. 1988, 10, 137.

41. Blázquez, G.; Hernáinz, F.; Calero, M.; Ruiz-Núñez, L. F.; Process Biochem. 2005, 40, 2649.

42. Fagundes-Klen, M. R.; Veit, M. T.; Silva, E. A.; Bergamasco, R.; Martins, T. D.; Canevesi, R. L. S.; Acta Science, Technology and Management 2011, 33, 439.

43. Aditya, G. V. V.; Pujitha, B. P.; Babu, N. C.; Venkateswarlu, P.; Korean J. Chem. Eng. 2012, 29, 64.

44. Saqueto, K. C.; Rosolino, R. M.; Machado, A. M. R.; Salvador, N. N. B.; Resumos do XLVI Congresso Brasileiro de Química, Salvador, Brasil, 2006.

45. Kotrba, P.; Machova, M.; Macek, T.; Microbial biosorption of metals (2011), doi: 10.1007/978-94-007-0443-5.

46. Mesquita, L. M. S.; Pinto, G. S.; Cunha, C. D.; Leite, S. G. F.; Methodus: revista científica e cultural 2001, 3,131.
47. Romera, E.; Gonzáles, F.; Ballester, A.; Blázquez, M. L.; Muñoz, J. A.; Bioresour. Technol. 2007, 98, 3344.

48. Tedesco, C.; Trabalho de Conclusão de Curso, Universidade de Passo Fundo, Passo Fundo, Brasil, 2010.

49. Giles, C. H.; Macewan, T. H.; Nakhwa, S. N.; Smith, D.; J. Chem. Soc. 1960, 3973

50. Manosalva, L. I. A.; Tese de Doutorado, Universidad de La Frontera, Temuco, Chile, 2008.

51. Góes Filho, L. S.; Tese de Doutorado, Pontifícia Universidade Católica do Rio de Janeiro, Rio de Janeiro, Brasil, 2010.

52. Souza, R. F.; Faquin, V.; Torres, P. R. F.; Baliza, D. P.; Rev. Bras. Cienc. Solo 2006, 30, 975

53. Doshi, H.; Seth, C.; Ray, A.; Kothari, I. L.; Curr. Microbiol. 2008, 56, 246. 